\title{
Laser metrology concept consolidation for NGGM
}

\author{
ICSO 2018
}

K. Nicklaus, S. Cesare, L. Massotti, L. Bonino, S. Mottini, M. Pisani and P. Silvestrin

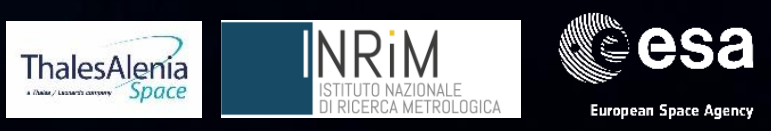




\section{Introduction}

GRACE (2002-2017)

- GER - US collaboration

- Measurement of time variable gravity field

- Microwave instrument

- 1 cm EWH@ 900 km

\section{GRACE FO (2018 - )}

- GER - US collaboration

- Microwave instrument as baseline

- Laser ranging interferometer as demonstrator
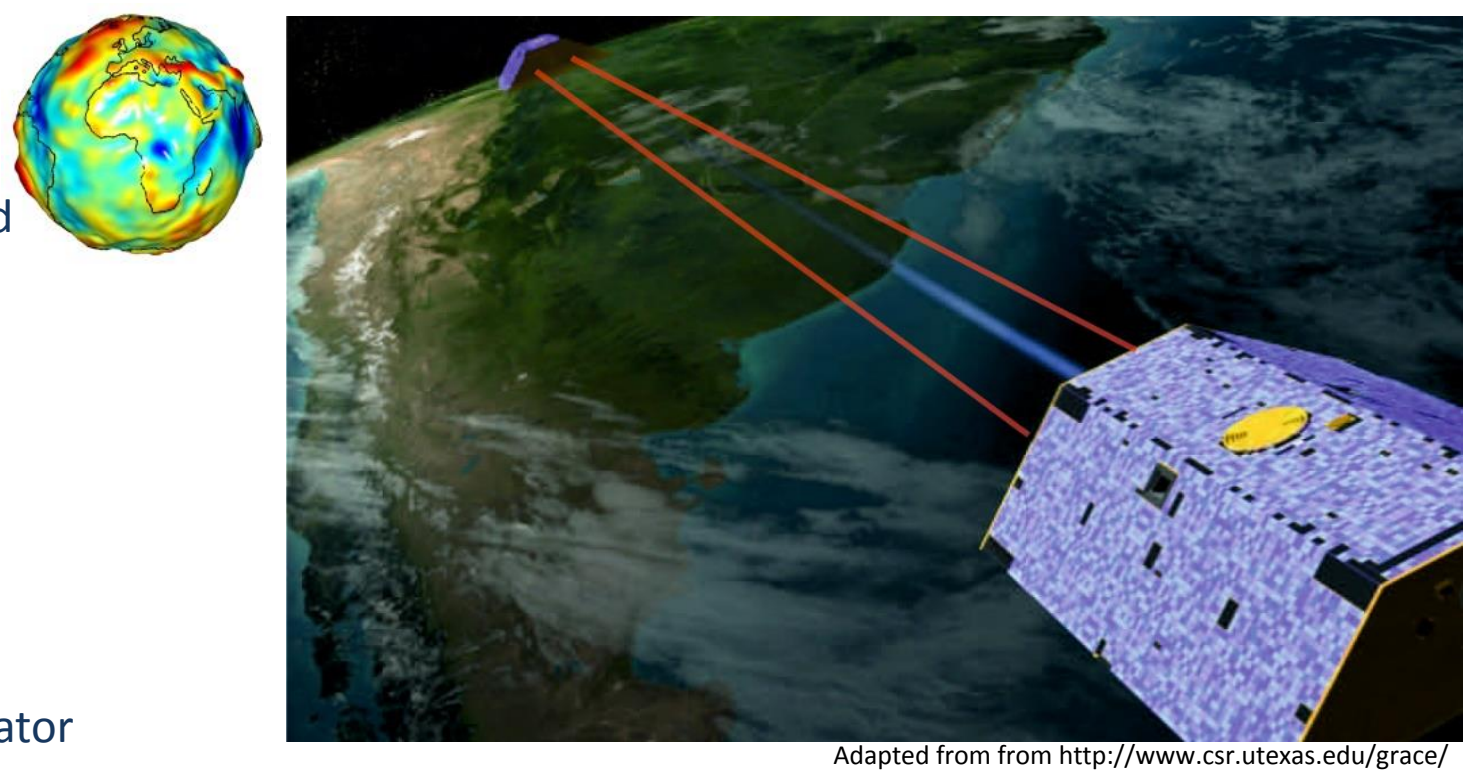

\section{NGGM}

- ESA Mission concept

- Laser Metrology Instrument

- 2 pairs of satellites at different inclinations

- 1 cm EWH@150km

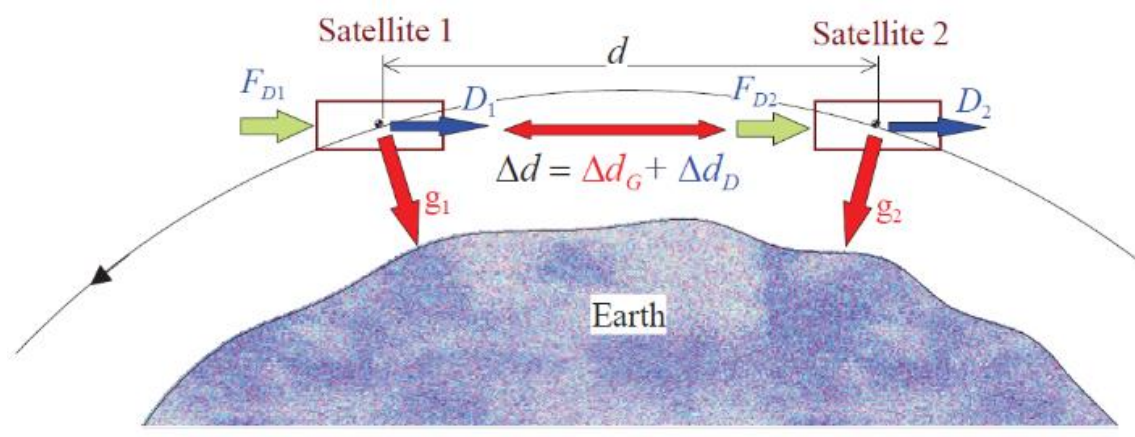




\section{LMI Overview}

- The LMI is an inter-satellite heterodyne interferometer, measuring the rel. Velocity changes of two satellites $100 \mathrm{~km}$ apart

- Two Schemes for the LMI are under consideration:

1. Transponder Scheme, with two active $S / C$ instrument parts, with GFO heritage

2. Retroreflector Scheme, with one active one passive $\mathrm{S} / \mathrm{C}$ instrument part, from former NGGM studies

- Both may be implemented in on and off-axis configuration

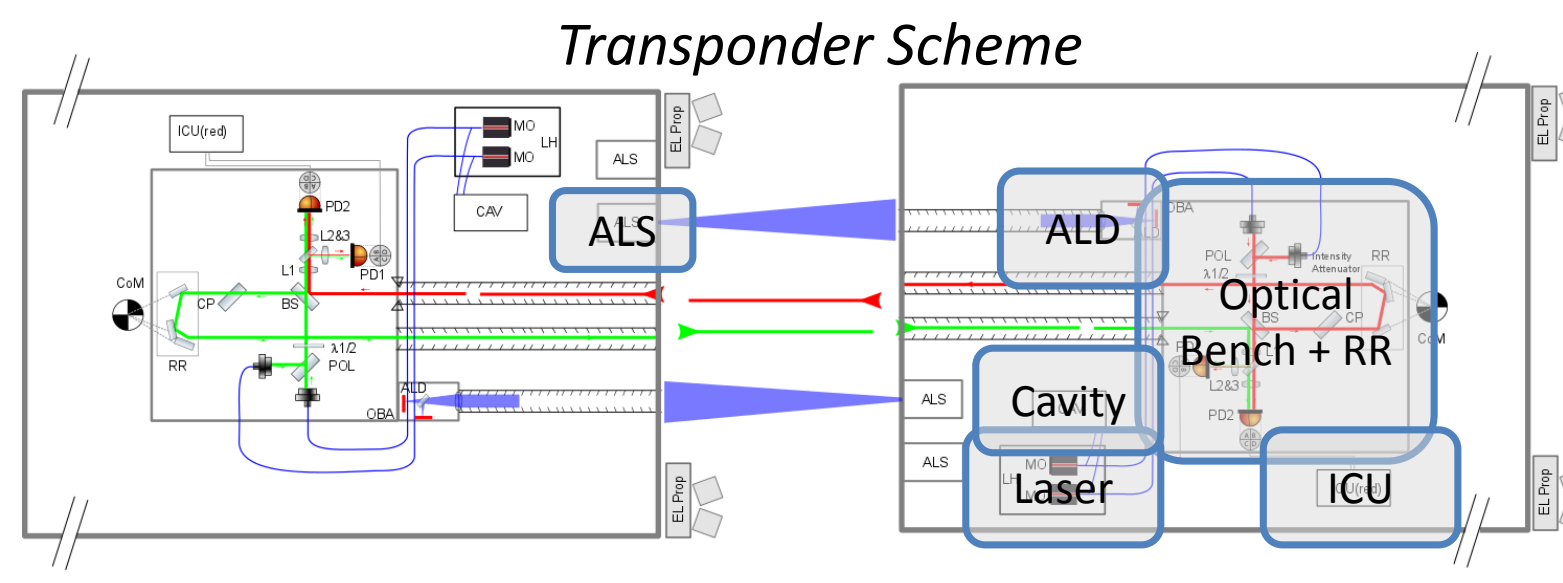

Retroreflector Scheme
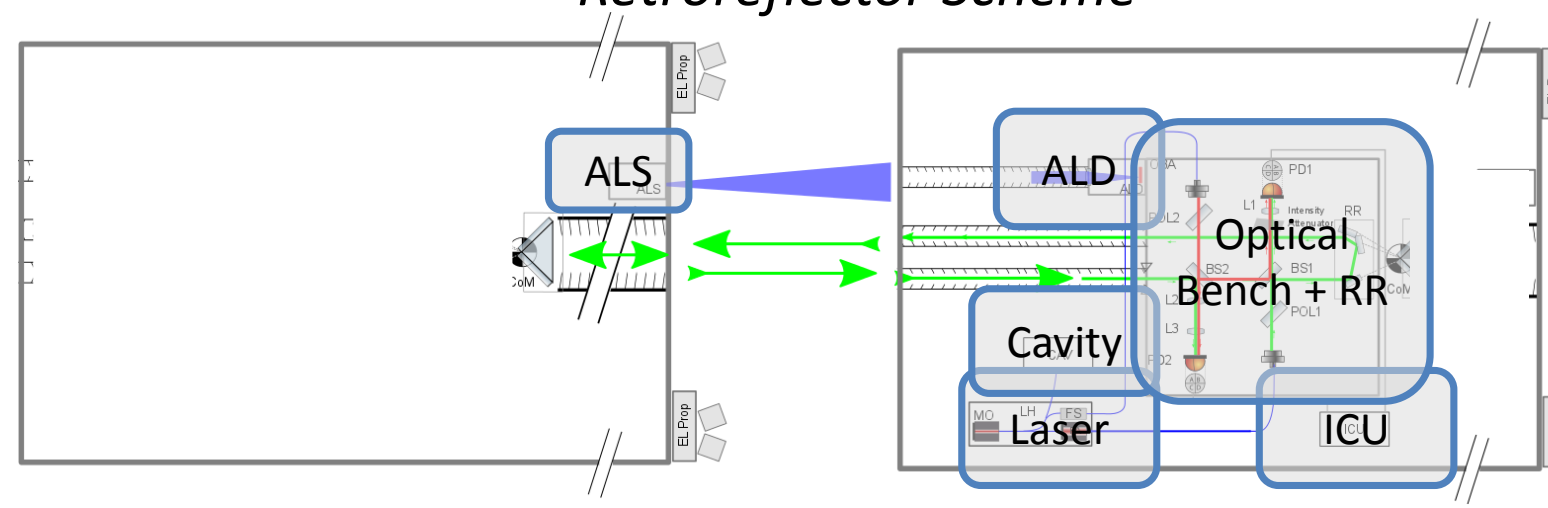


\section{Key requirements}

- Ranging noise requirement

$$
\tilde{x}_{\text {thy }}=20 \frac{n m}{\sqrt{H z}} x \sqrt{1+\left(\frac{10 m H z}{f}\right)^{2}} \text { for } 1 \times 10^{-4} \mathrm{~Hz}<f<1 \mathrm{~Hz}
$$

- Ranging noise goal

$$
\tilde{x}_{\text {goal }}=10 \frac{n m}{\sqrt{H z}} x \sqrt{1+\left(\frac{10 \mathrm{mHz}}{f}\right)^{2}} \text { for } 1 \times 10^{-4} \mathrm{~Hz}<f<1 \mathrm{~Hz}
$$

- Thermal environment

- Cavity $0.01 \mathrm{~K} / \sqrt{ } \mathrm{Hz}$ x NSF(f) (from GOCE)

- Retroreflector $0.1 \mathrm{~K} / \sqrt{ } \mathrm{Hz} \times \mathrm{NSF}(\mathrm{f})$

- Optical Bench $0.3 \mathrm{~K} / \sqrt{ } \mathrm{Hz}$ x NSF(f)

- Satellite Pointing error

$<20 \mu \mathrm{rad}$ (simulation shows $<2 \mu \mathrm{rad}$ )

- Satellite Pointing noise

- $2 \mu \mathrm{rad} / \sqrt{\mathrm{Hz}} \times \mathrm{NSF}(\mathrm{f})$

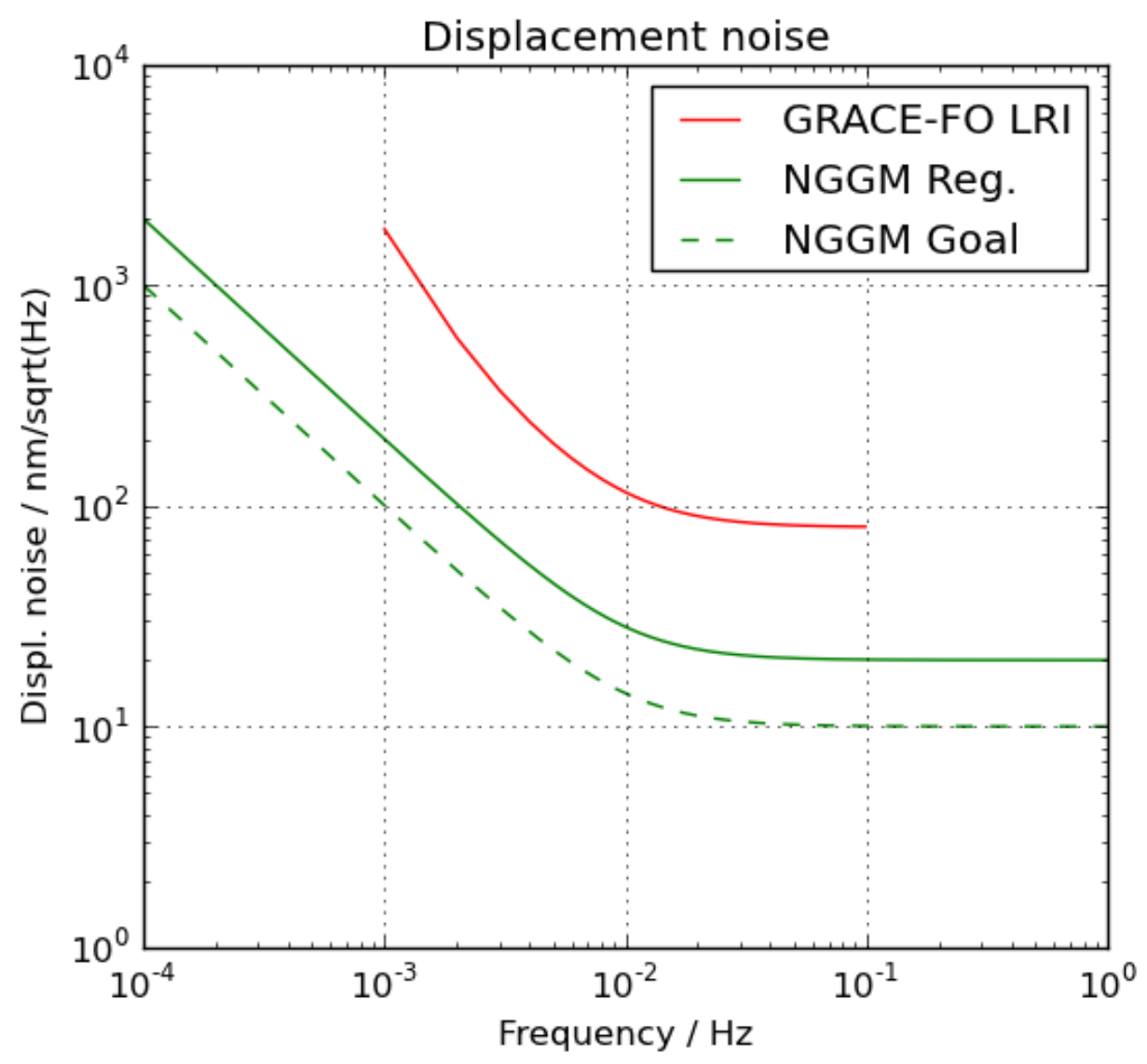

- GFO: $300 \mu \mathrm{rad} / \sqrt{ } \mathrm{Hz} \times \mathrm{NSF}(\mathrm{f})$ 


\section{On- and Off-axis configurations}

\section{Transponder Scheme}

For both schemes, the offaxis configurations are considered superior due to

- No need to direct access of CoM

- less criticality with respect to polarisation

- straylight / multipath reflections

- complexity of setup/number of optical elements in the beam path

- complexity of operation.
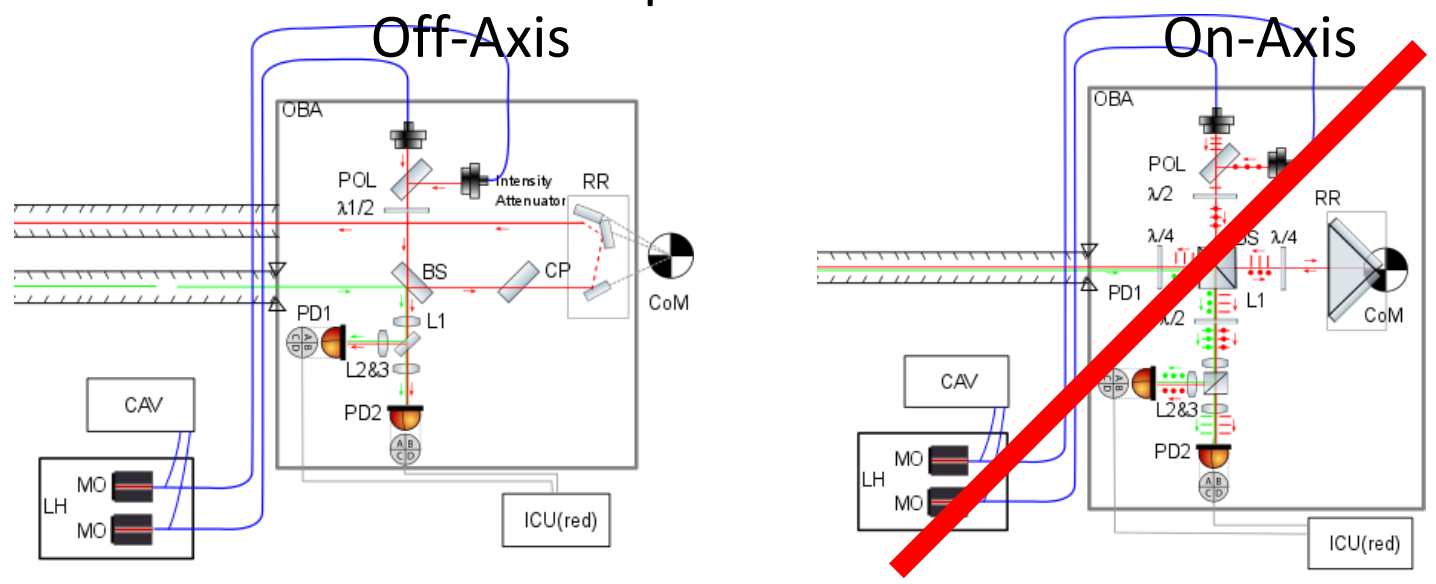

Retroreflector Scheme

As design guideline:

- Minimize the number of optical components in measurement path

- Benefit from common mode paths in beam delivery as much as possible
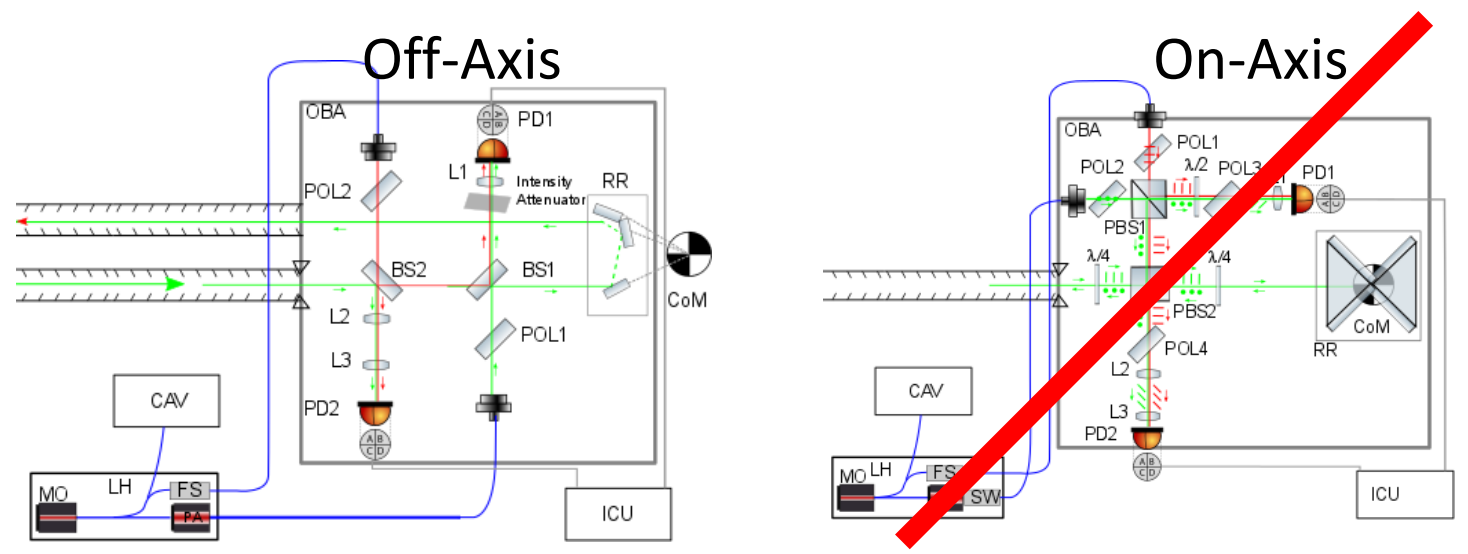


\section{Transponder Scheme}

\section{Off-Axis ("Racetrack") configuration}

- Based on racetrack design applied on GRACE Follow-On

- Dedicated Link Acquisition system on both spacecraft

- Minimizes optical elements in beam path, only common delivery beam paths

- Redundancy on subsystem level (Lasers, Photoreceivers, Cavity, electronics)

\begin{tabular}{|l|l|}
\hline$P_{\text {laser }}$ & $25 \mathrm{~mW}$ \\
\hline$P_{\text {rec }}$ & $1-3 \mathrm{nW}$ \\
\hline$P_{\text {el }}$ & $65 \mathrm{~W}$ \\
\hline Mass & $43 \mathrm{~kg}$ \\
\hline
\end{tabular}

- Two Satellite configurations with respect to el. propulsion
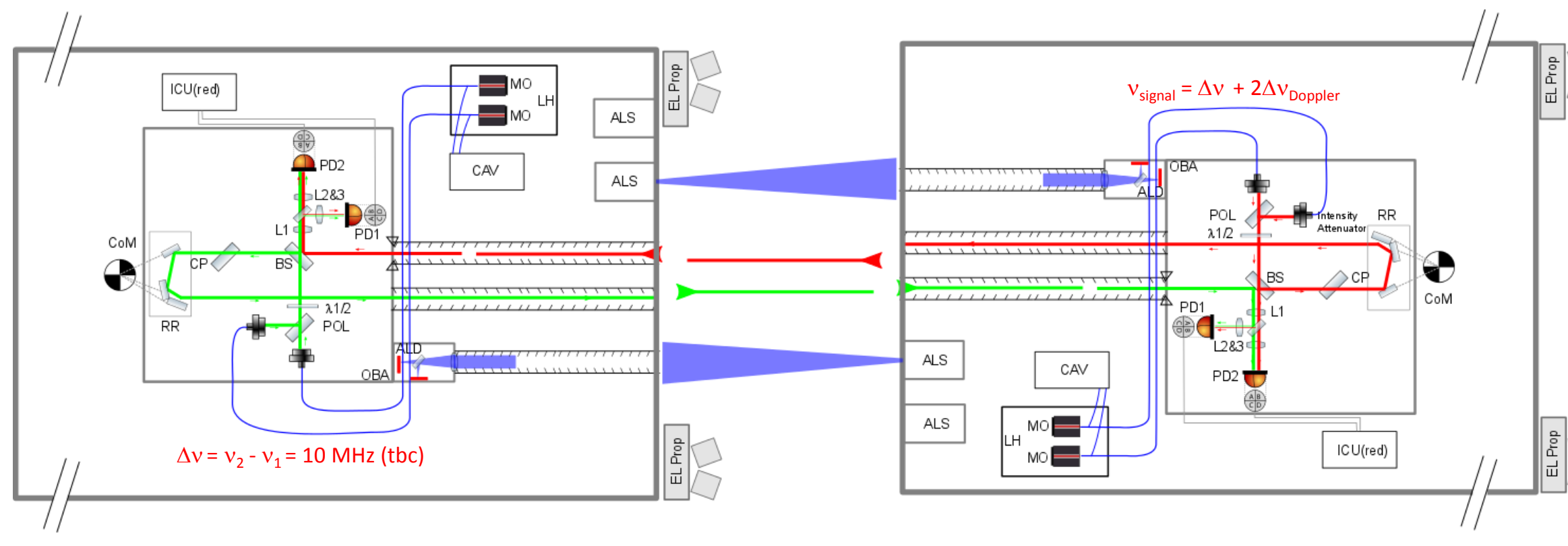


\section{Retroreflector Scheme}

\section{Off-Axis ("Racetrack") configuration}

- Based on former NGGM studies

- Dedicated Link Acquisition system on both spacecraft

- Reference photoreceiver for non-common delivery beam path noise.

- Full redundancy on instrument level

- Identical configuration for both satellites

- Requires significant suppression of straylight and multipath reflections on optical bench

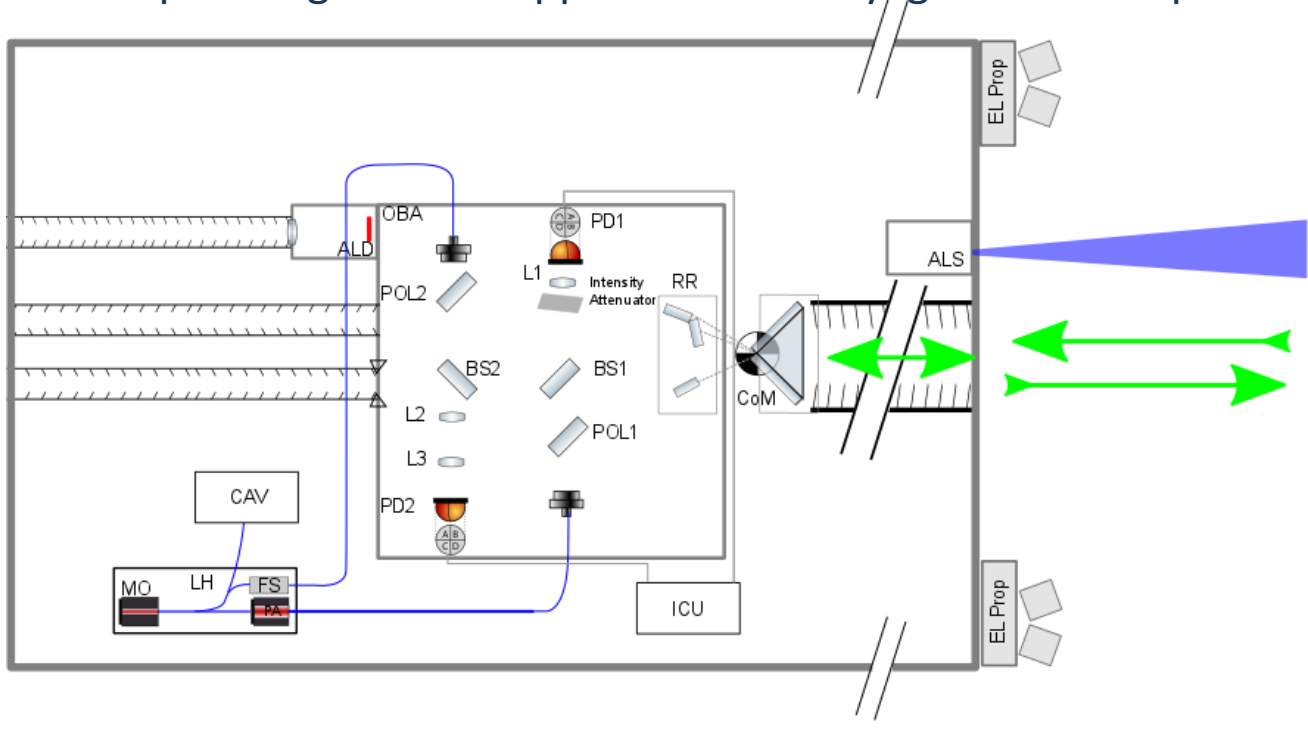

\begin{tabular}{|l|l|}
\hline$P_{\text {laser }}$ & $500 \mathrm{~mW}$ \\
\hline$P_{\text {rec }}$ & $10-35 \mathrm{pW}$ \\
\hline$P_{\text {el }}$ & $96 \mathrm{~W}$ \\
\hline Mass & $45 \mathrm{~kg}$ \\
\hline
\end{tabular}

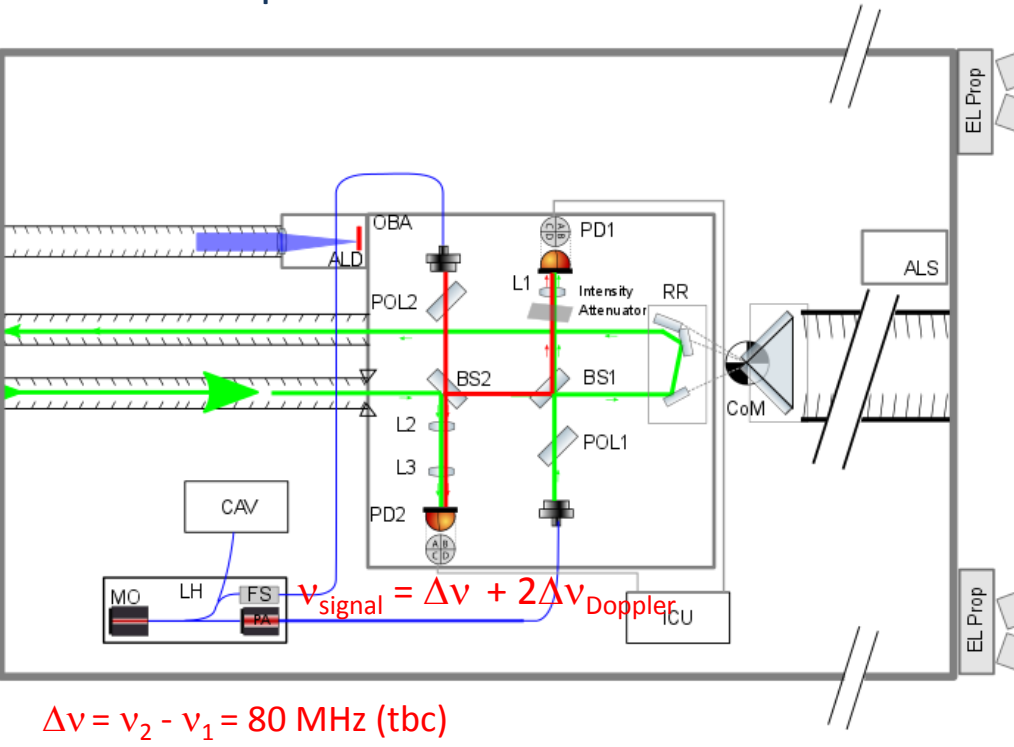




\section{Performance Analysis - ranging noise}

- Laser frequency noise

- Photoreceiver noises (Shot noise, El. Noise, Power Noise, Dark current)

- RR thermal noise

- OBA thermal noise

- SST path noise

- Delivery path common mode noise

- non common mode deliver path noise

- RR coupling with SC rotation

- OBA coupling with SC rotation

- Coupling of wavefront planarity with beam pointing error

- Satellite CoM Stability

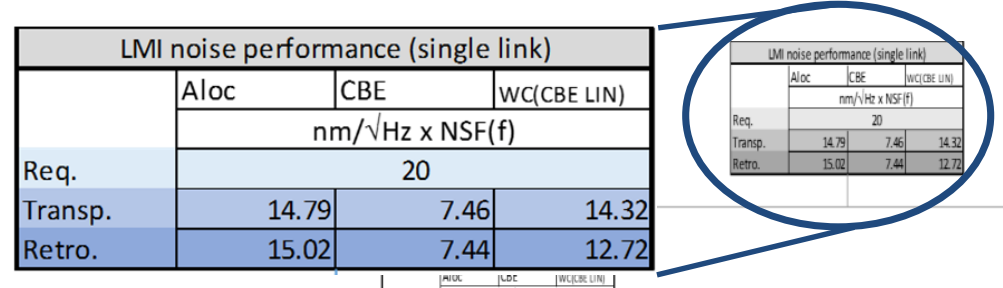

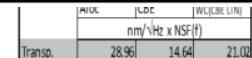

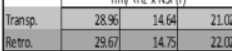

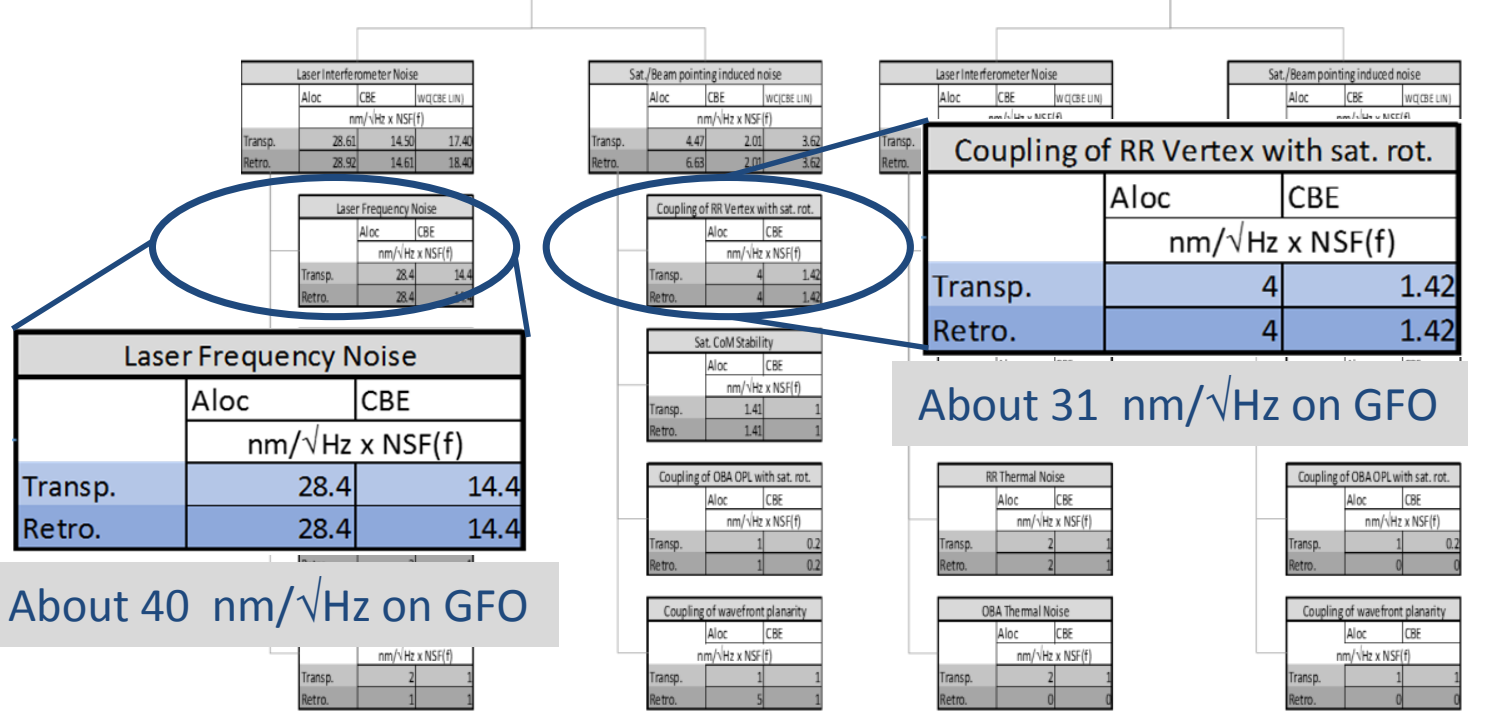

Laser frequency noise is dominating ranging noise 


\section{STI LMI Units and TRL assessment}

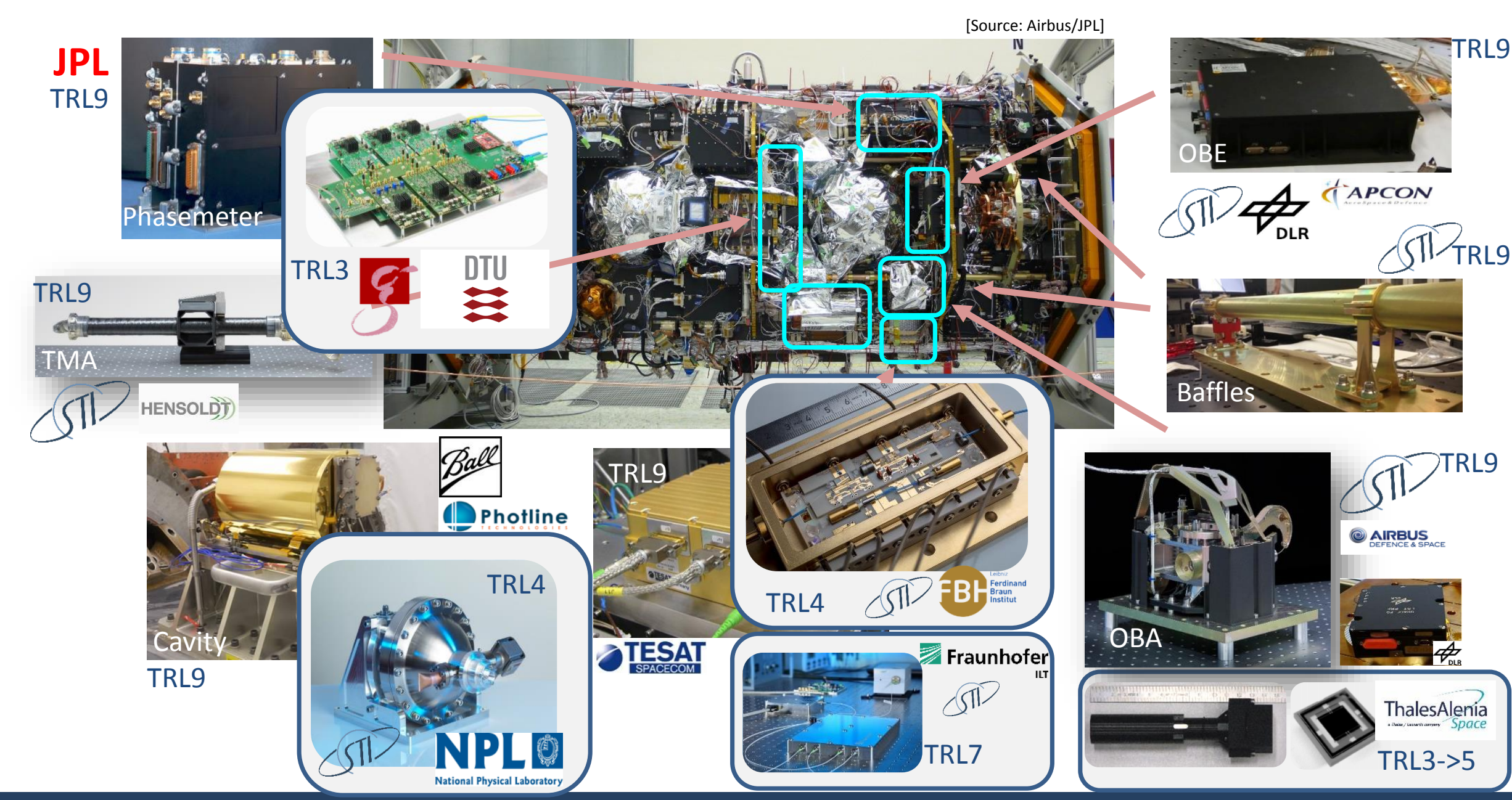




\section{Seed Laser Building Block}

FBH microintegrated diode laser technology has the potential be a "seed laser building block" for many missions

- $500 \mathrm{~mW}$ output power

- <100 kHz linewidth

- approx. 10 \% EO efficiency

- 630-1180 nm wavelength range

- DFB/ECDL/ECDL-ROF with amplifier

Application in:

- Science (LISA, optical clocks),

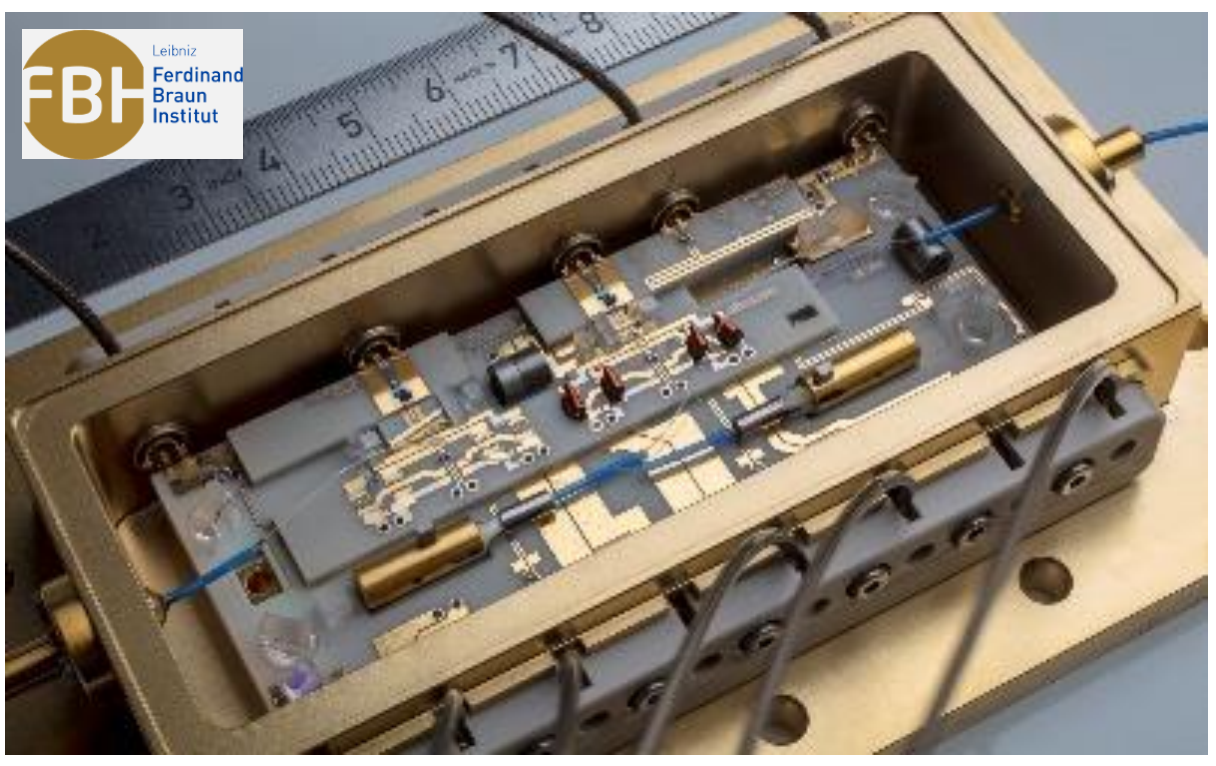

- EO (NGGM, LIDAR seeder,...)

- Optical communication

Developments running for
- LISA
- NGGM
- DWDM
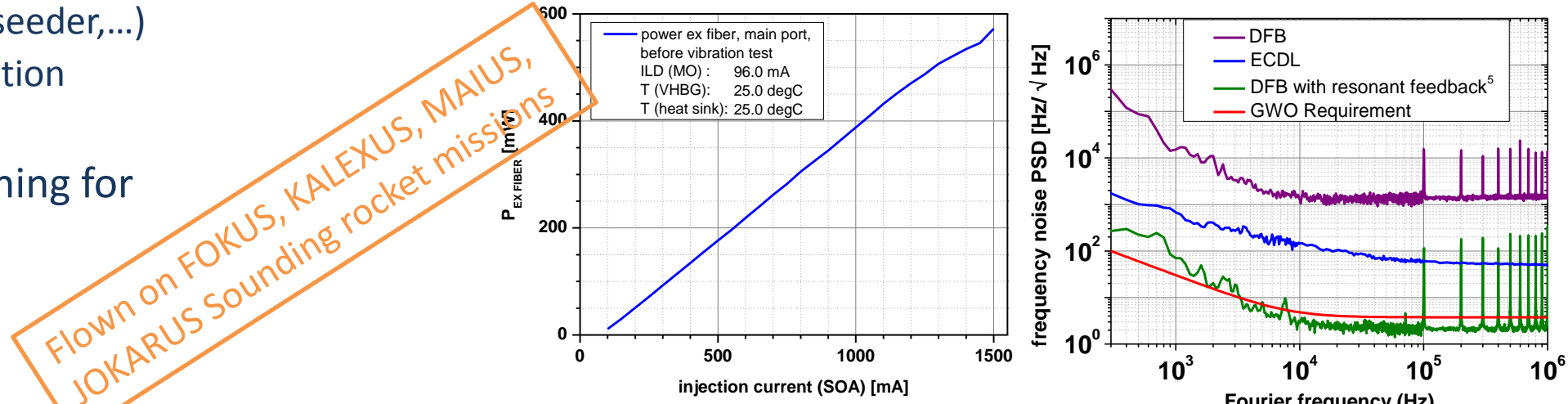


\section{Link Acquisition - Transponder Scheme}

- Initial Pointing uncertainty: +/- 3 mrad

- Initial frequency offset: $+/-1 \mathrm{GHz}$

- 3 mrad to 100 urad (ALS \& ALD)

- $S / C 1$ (master) -> rotate to $S / C 2$

- $\mathrm{S} / \mathrm{C} 2$ (slave) -> rotate to $\mathrm{S} / \mathrm{C} 1$

- From $100 \mu \mathrm{rad}$ to $<50 \mu \mathrm{rad}$

- Slave frequency sweep (transp. only)

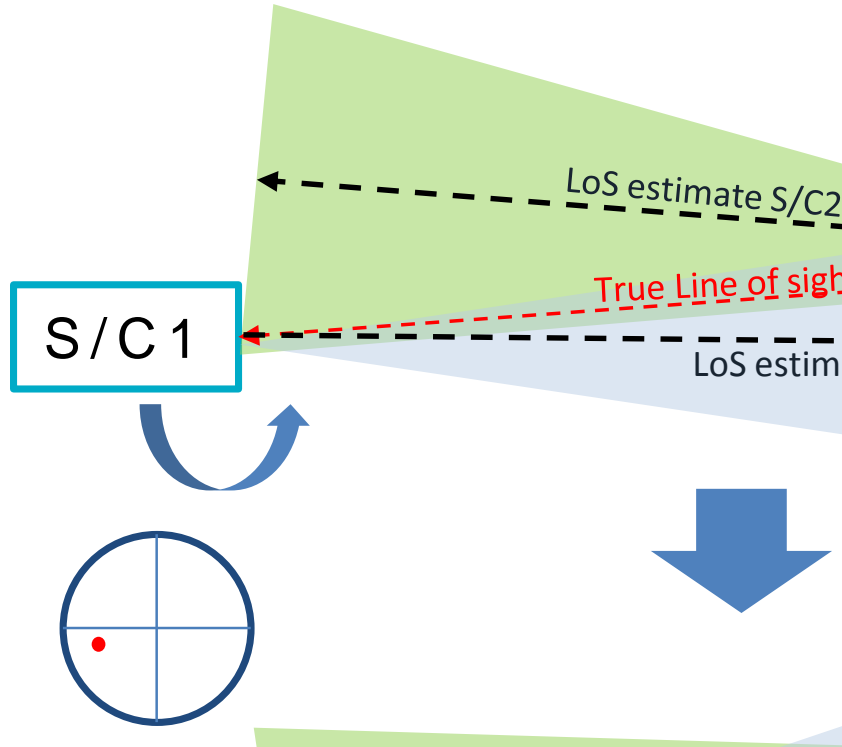

- use of DWS

- Turn of ALS \& ALD 


\section{Link Acquisition - Retroreflector Scheme}

- Initial Pointing uncertainty: +/- 3 mrad

- $3 \mathrm{mrad}$ to $100 \mu \mathrm{rad}$ (ALS\&ALD)

- S/C 1(active) -> rotate to S/C 2

- From $100 \mu$ rad to $<50 \mu \mathrm{rad}$

- use of DWS

- Turn of ALS \& ALD

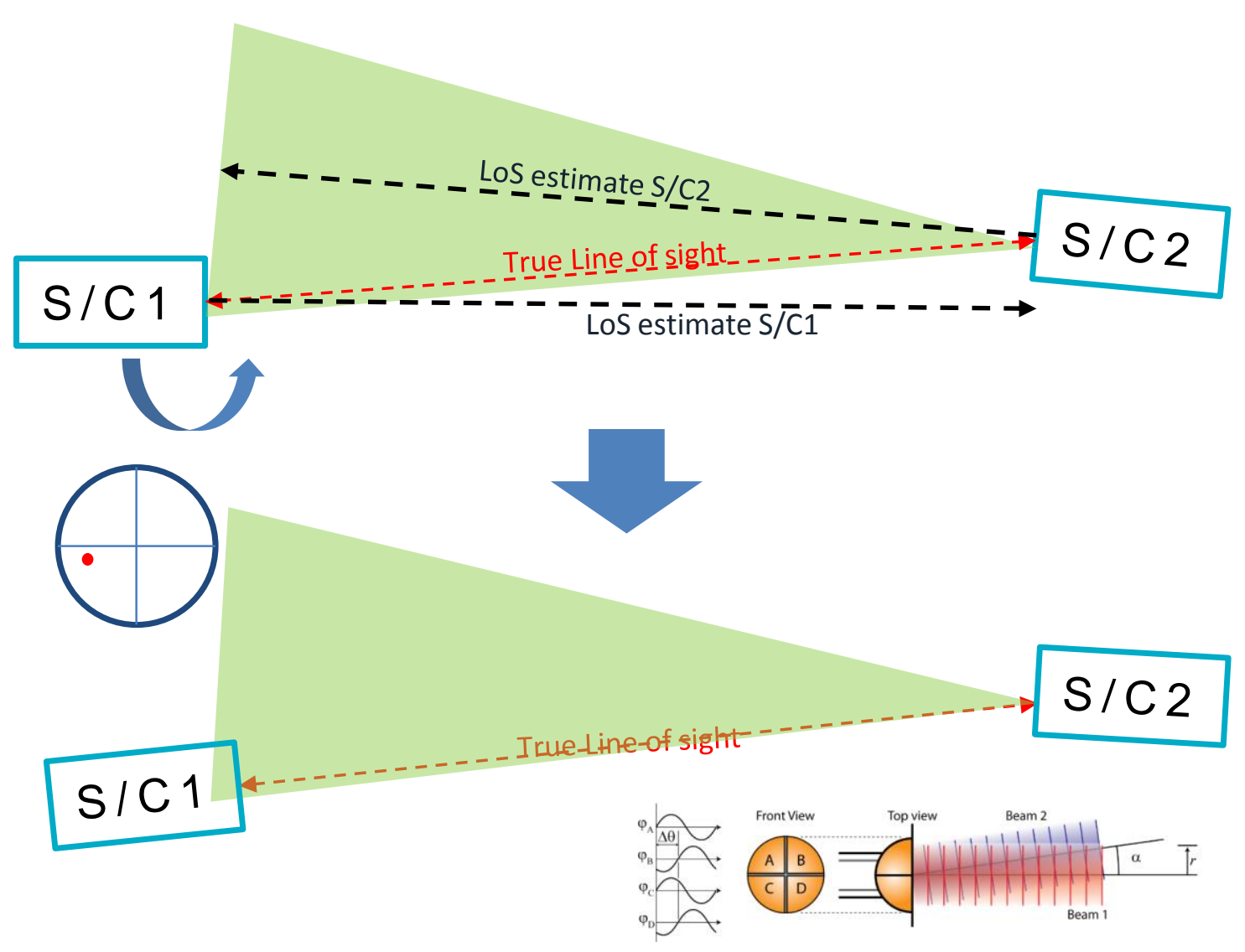




\section{Summary}

- Two concepts for the Laser metrology Instrument on NGGM are under investigation

- Off-axis transponder concept, derived from and flight proven on GFO, with dedicated link acquisition system

- Off-axis retroreflector concept, potentially allowing simpler satellite configuration, easier link acquisition - low received signal situation to be analysed further

- Both concepts are still considered viable options for NGGM

- Developments for ECDL Laser and fiber amplifier are ongoing

- Breadboarding of the optical bench of the retroreflector concept is planned.

\section{Thank you very much for your attention.}
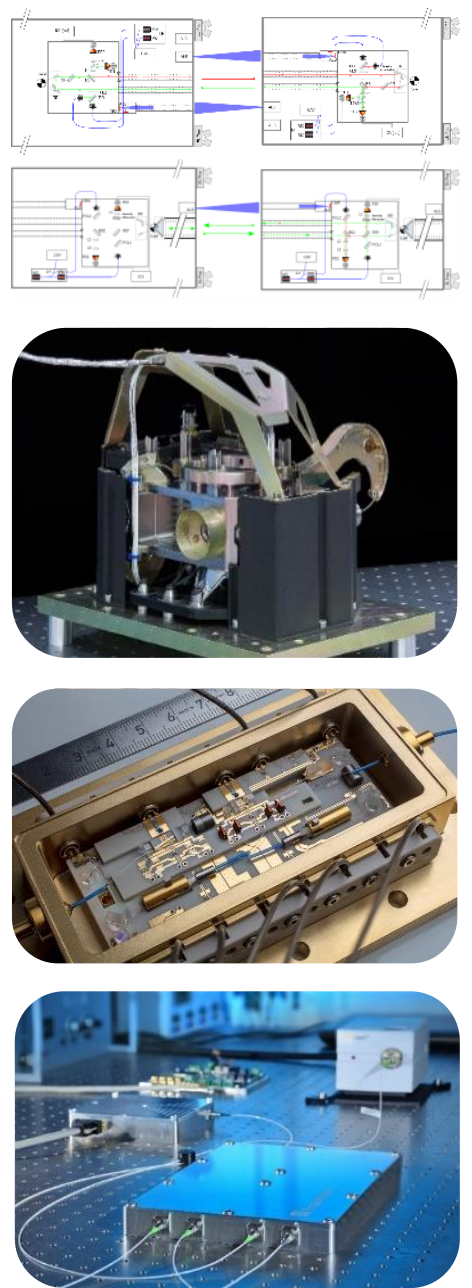


\section{Launch your visions}

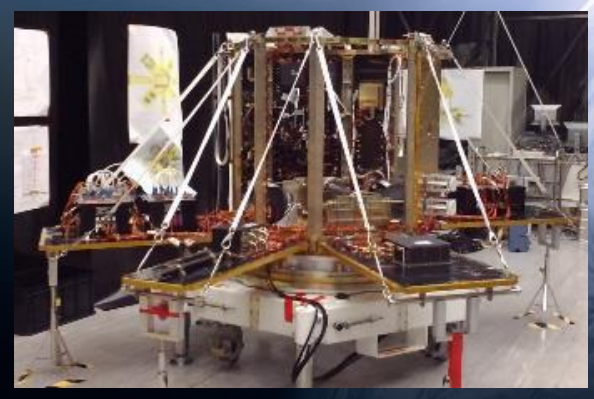

Small Satellites

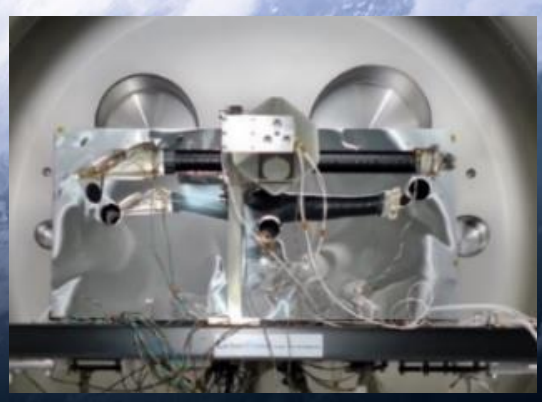

Optical Instruments

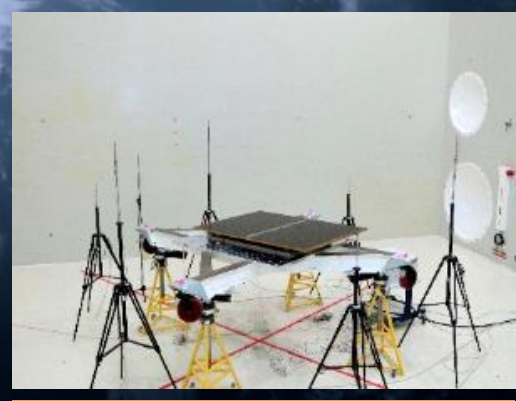

Satellite Equipment

Thank you very much for your attention. 